\title{
Evolución del proyecto del Salto del Jándula. Proceso de integración de la central hidroeléctrica en el cuerpo de presa
}

\author{
Evolution of the Salto of Jándula project. Process of integration \\ of the hydroelectric power station in the dam
}

\author{
N. Carbajal-Ballell $^{(*)}$
}

\section{RESUMEN}

El Salto del Jándula es una de las obras civiles paradigmáticas del siglo XX. Su característica central hidroeléctrica adosada al cuerpo de presa la ha convertido en referencia indiscutible entre arquitectos, ingenieros o historiadores. Y esta mirada poliédrica no es casual, pues desde su inicio se planteó como un proyecto multidisciplinar ligado a grandes planes territoriales. El riego en el valle del Guadalquivir y un ambicioso proyecto de canalización del río entre Córdoba y Sevilla fueron las razones por la que esta obra se llevaría a término, mas el origen de la singular solución que caracteriza su aprovechamiento hidroeléctrico es menos conocido siendo en realidad un resultado decantado a lo largo de sucesivos proyectos.

Palabras clave: Central hidroeléctrica, Fernández-Shaw, futurismo, Jándula, Mendoza, Mergemor, presa, salto.

\section{ABSTRACT}

The Salto of Jandula is one of the paradigmatic civil works of the 2oth century. Its characteristic hydroelectric power station attached to the dam has made it an indisputable reference among architects, engineers and historians. And this polyhedral look is not accidental, since from its beginning, it was proposed as a multidisciplinary project related to large territorial plans. Irrigation in the Guadalquivir valley and an ambitious project to channel the river between Córdoba and Seville were the reasons why this work was built, but the origin of the unique solution that characterizes its hydroelectric use is less known and is actually a result decanted throughout successive projects.

Keywords: Hydroelectric power station, Fernández-Shaw, futurism, Jándula, Mendoza, Mergemor, dam.

(*) Universidad de Sevilla (España).

Persona de contacto/Corresponding author: ncarbajal@us (N. Carbajal-Ballell)

ORCID: http://orcid.org/oooo-0002-0423-2605 (N. Carbajal-Ballell)

Cómo citar este artículo/Citation: Carbajal-Ballell, N. (2019). Evolución del proyecto del Salto del Jándula. Proceso de integración de la central hidroeléctrica en el cuerpo de presa. Informes de la Construcción, 71(554): e294. https://doi.org/10.3989/ic.63725

Copyright: (c) 2019 CSIC. Este es un artículo de acceso abierto distribuido bajo los términos de la licencia de uso y distribución Creative Commons Reconocimiento 4.0 Internacional (CC BY 4.0). 


\section{ORIGEN DEL PROYECTO}

La naturalidad que aparentan las soluciones técnicas del Salto del Jándula parece indicar la existencia de un proyecto sencillo, de aquellas ideas que por su claridad, rotundidad e idoneidad se demuestran incontestables y toman forma de manera inmediata, pero la investigación realizada muestra sorprendentemente como al contrario, es consecuencia de un ingente esfuerzo proyectual, debido a una complejidad técnica notable y a la conjunción de múltiples y dispares intereses.

Su construcción dio comienzo en febrero de 1927 después de un dilatado proceso iniciado años atrás. En el plan de obras hidráulicas de 1902, figuraba con el número 103 la previsión de un embalse de $100 \mathrm{hm}^{3}$ en la misma ubicación del actual destinado fundamentalmente al riego en la margen izquierda del Guadalquivir (1). Poco después el plan de creación de regadíos de 1907 hacía mención a un gran pantano en las cercanías de Andújar, ubicado en la cerrada de La Charca del Fraile, paraje que ocupa el embalse. Un estudio que compendiaba no sólo la ubicación de las obras hidráulicas necesarias sino que las definía con aproximación recomendando tipos estructurales, perfiles o materiales a emplear (2). Estos cultivos serían regados desde embalses situados en la vertiente derecha de un Guadalquivir que también era objeto de estudios para hacerlo fácilmente navegable entre Córdoba y Sevilla. Es el caso del conocido proyecto de Mengemor que combinaba -situando una serie de pequeñas presas- el aprovechamiento hidroeléctrico con su navegabilidad mediante unas esclusas laterales (3). El objetivo de este salto sería por tanto favorecer la implantación del regadío en el valle y garantizar la navegabilidad del río en los periodos de mayores estiajes asegurando unos caudales mínimos para el funcionamiento de las centrales que incluía aquel proyecto y ayudaban a financiar tamaña empresa (4). Del mismo modo es indudable que eran necesarias nuevas centrales y nuevas líneas eléctricas que fueran vertebrando un sistema a escala territorial mayor que aquel derivado de su origen industrial y minero, para hacer frente a las crecientes demandas energéticas que eran ahora también de índole urbana (5). Y en cuanto a la singular central hidroeléctrica, se han señalado algunos precedentes tanto técnicos como formales que situaban el aprovechamiento adosado al muro, es el caso de presas como la de Gaitanejo en España, las de Coolidge, La Boquilla o Don Pedro en América o Santa Chiara y Belle Île en Europa, incluso de otras menores vinculadas a molinos hidráulicos en siglos pasados, pero también de ideas presentes en la propia obra de Casto Fernández-Shaw -arquitecto coautor del proyecto- como la de su Monumento a la Civilización (6)(7).

\section{PROYECTO COMPLEMENTARIO DEL DE CANALIZACIÓN Y APROVECHAMIENTO DE ENERGÍA DEL GUADALQUIVIR, MEDIANTE NUEVOS APROVECHAMIENTOS REGULARIZADORES DE SU RÉGIMEN EN EL JÁNDULA Y EL RÍO DE LAS YEGUAS. 20 DE ENERO DE 1921}

Aparece así vinculada al proyecto de canalización del Guadalquivir que redacta Carlos Mendoza y ligado al de otro aprovechamiento hidroeléctrico en el Burcio de Valquemado. Un documento presentado dos años después de aquel que junto a la memoria, adjunta sendos anejos, uno de Planos y otro titulado Tarifas y Presupuestos con una estimación del coste previsto para las obras y una tabla con las que serían las tarifas aplicables para los riegos y los usos industriales.

La colección de seis planos -firmados por Mendoza - trataba de ofrecer de manera muy general una imagen gráfica de ambos embalses. El $\mathrm{n}^{0} 1$ es un plano de la cuenca titulado Salto de la Charca del Fraile-Río Jándula que incluye una sección muy esquemática -a escala 1:10oo- del cuerpo de presa. En ella se acota en $44,5 \mathrm{~m}$ su altura dejando un margen de medio metro con el nivel máximo normal del embalse, también su ancho en coronación de $4 \mathrm{~m}$, la inclinación del paramento aguas abajo y el nivel de estiaje del río. El segundo, con idéntico título en su carátula, es una planta de la presa. Sin escala pero sobre un topográfico de la cerrada, indica el eje y el radio del arco que traza y puede apreciarse cómo la central está asentada en un lateral sobre la línea de nivel +290,00 (Figura 1). El tercero es la Sección transversal del emplazamiento a escala 1:1000 con el alzado aguas abajo de la presa y grafiado en trazo discontinuo la línea de su coronación una vez se ampliara a $60 \mathrm{~m}$. No representa la central hidroeléctrica pero nos permite observar un labio de vertido sobre su coronación. El cuarto (Figura 2) -carente también de escala- muestra una planta y una sección de la central hidroeléctrica, una edificación convencional y exenta a un cuerpo de presa que se prevé ampliable como puede comprobarse en otra transversal que corta por la toma de aguas. Los planos 5 y 6 son relativos al Salto del Burcio de Valquemado e igual de esquemáticos.

La memoria en un sintético apartado -Edificio- define en pocas líneas lo concerniente a ella: «Paralelamente al cauce del río y adosado a la presa, se levantará el edificio para la casa de máquinas. El piso de esta estará a unos $6 \mathrm{~m}$ sobre el nivel de estiaje, y las puertas y ventanas estarán de tal forma que el agua, durante las crecidas, no pueda penetrar en su interior». «La anchura interior del edificio será de $10 \mathrm{~m}$ y se dispondrá en el sentido longitudinal de manera que pueda prolongarse cuando sea necesario aumentar las máquinas instaladas, una vez aumentada hasta $60 \mathrm{~m}$ la altura de la presa».

En este momento se piensa en una edificación a pie de presa y observando cómo está asentada en la margen derecha comprendemos que el labio de vertido en la coronación esté desplazado hacia la izquierda tratando de alejar de la misma el torrente de las aguas, algo sorprendente pues en realidad en el futuro la carretera de Los Escoriales a La Lacha llegará por la margen opuesta y por tanto, no se podría cruzar hasta ella pues como decimos, la presa presenta un esquema que permitiría el desbordamiento por coronación similar en su disposición, aunque menos novedoso en su formalización, al que se emplearía más tarde para la cercana de Encinarejo de los mismos autores. Toda su organización habría de ser longitudinal (por la disposición en serie prevista para los grupos hidroeléctricos), adaptándose no sólo a las necesidades de la futura ampliación sino también aunque no lo mencione, al escaso espacio en el seno de la cerrada. Estaría próxima a la presa, a unos $15 \mathrm{~m}$ de distancia, pero no adosada como mencionan y en todo caso no integrada en el faldón del macizo.

Nada se indica tampoco sobre sus características constructivas salvo los apuntes acerca de la necesidad de estanquidad de sus huecos y de ese ancho de la edificación estimado. Se trata en definitiva de una sencilla nave asentada sobre una losa de cimentación extendida sobre el lecho rocoso y acodada en su nivel inferior sobre un largo muro de hormigón levantado al 


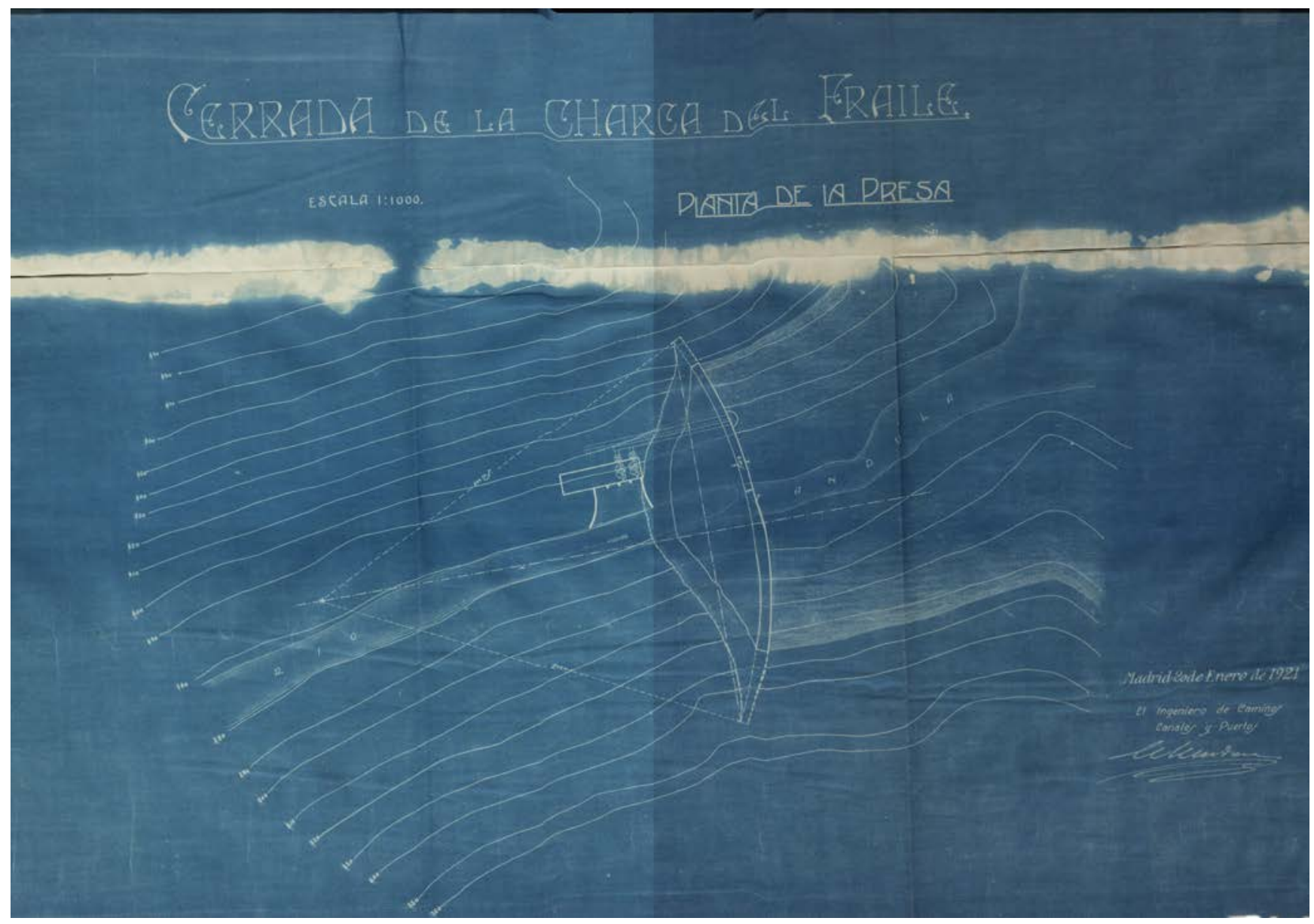

Figura 1. Plano $n^{0} 2$ - Planta de la presa. Fuente: Archivo de la Fundación Endesa, Sevilla.

cajear la roca; de amplios ventanales -once en cada una de las largas fachadas y dos sobre ambos testeros- y cubierta a dos aguas. De unos 38,5 m de longitud, 11 de ancho y 15 de alto, dividida en dos salas, una destinada a los generadores y la otra de menor tamaño, a los transformadores. Contaría con un puente grúa y dejaría el espacio suficiente a disposición de futuras incorporaciones de nuevos grupos hidroeléctricos. La presencia de este puente grúa hace suponer una construcción capaz de soportarlo -quizá en hormigón pero probablemente de gruesos muros mampuestos- y una cubrición con cerchas de acero o armaduras de madera.

No se indican los accesos aunque probablemente se situarían sobre su costado derecho -por el que también llegan las conducciones- ya que expulsaría las aguas turbinadas al río por el izquierdo, pero no directamente, pues la distancia que la separaría del cauce haría conveniente un amplio cuenco de descarga. Constaría de tres niveles, el superior donde estarían instalados los grupos, uno intermedio donde acometerían las tuberías a las turbinas y otro de apoyo sobre el cauce, abovedado, por donde se expulsarían las aguas turbinadas. Este último no parece ser visitable. Las dos plantas superiores habrían de tener accesos independientes pues no disponen de escaleras que permitan alguna comunicación interior. El hecho de que las turbinas evacuaran verticalmente los potentes chorros de agua podría justificar la presencia de esa gran losa que mitigara los efectos de la socavación en los cimientos de la central. Pero en realidad todo ello es cuestionable pues la escasa documentación gráfica que aporta el proyecto es contradictoria y no permite extraer conclusiones precisas de todos los aspectos no justificados en la memoria. Mientras la planta sitúa la edificación a $10 \mathrm{~m}$ sobre el nivel del río y bien distanciada de él, la sección de la central la muestra bañada por sus aguas, solución más adecuada al hecho de que las turbinas expulsaran hacia abajo, absorbiendo esa fuerza la propia masa acuosa del río al estar inmersa en ella. En cualquier caso resulta difícil pensar que un cauce seco o regulado como aquel, el agua pudiera en condiciones de normalidad los niveles supuestos.

Podemos imaginarla esbelta y elegante como otras construcciones industriales del momento, edificaciones que proliferan entonces por el territorio y presentan características constructivas comunes, con sus grandes cristaleras que inundan de luz esos modernos espacios de trabajo ocupados por máquinas. Similar a otras instalaciones de carácter hidroeléctrico como la cercana de Mengíbar construida poco antes, obra del arquitecto Antonio Palacios también para la compañía Mengemor, cuya influencia formal parece innegable. Serena, anónima, discretamente acomodada al pie de la ladera y completamente ajena al carácter expresionista que le terminaría dando identidad propia. En definitiva, un diseño sustancialmente distinto de aquel que caracterizará a la presa del Jándula para la historia, un proyecto cuyo único elemento común era la elección del lugar para establecer el muro. Es una presa asimétrica y de menor envergadura, la central hidroeléctrica es una edificación convencional y exenta en una margen del río, las aguas se alivian por coronación y el torreón de maniobras es semicilíndrico y adosado a un costado y no el castrense que hoy la remata, mostrando todas estas características 


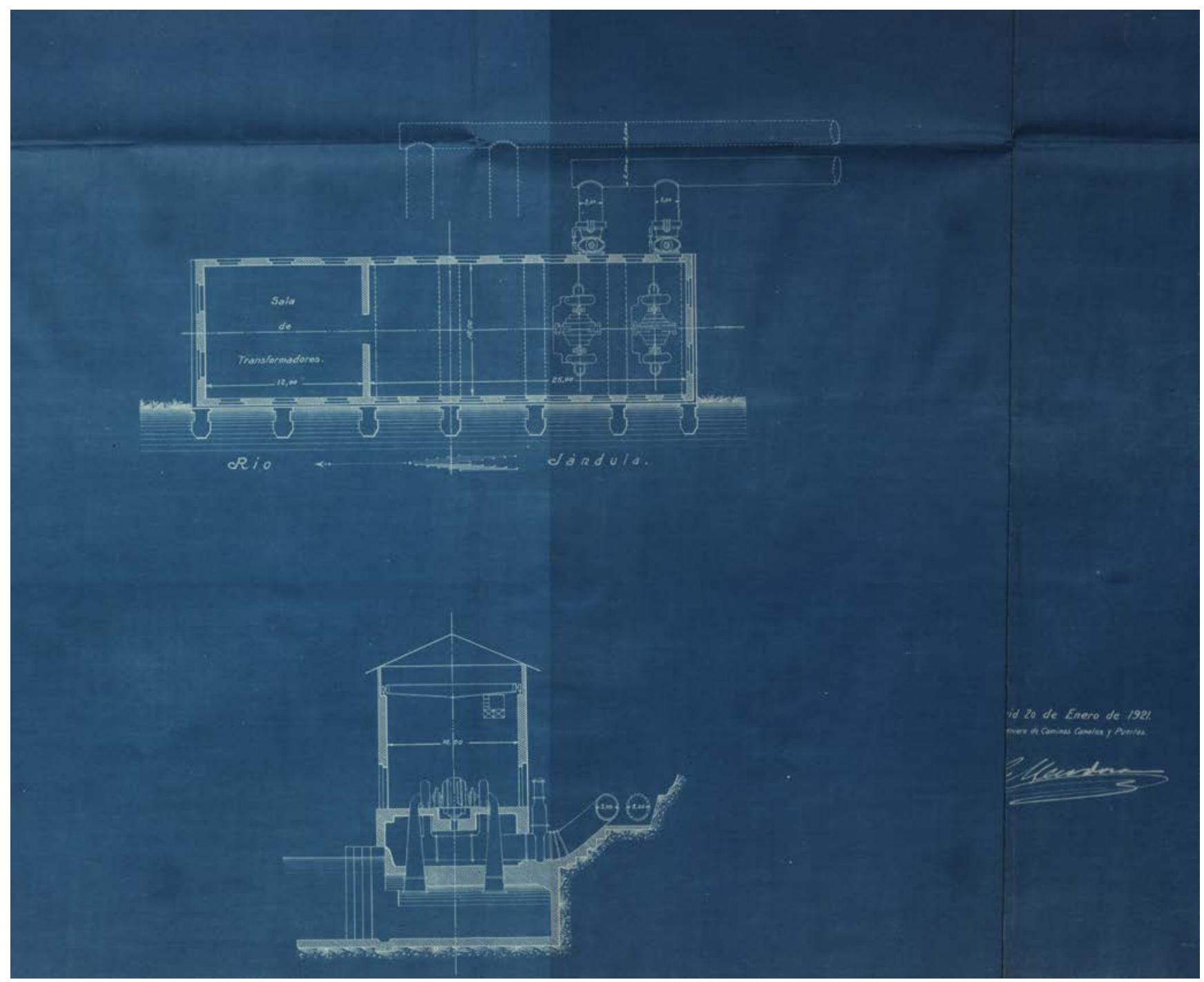

Figura 2. Detalle del plano $n^{\circ} 4$ - Planta y sección de la central hidroeléctrica. Fuente: Archivo de la Fundación Endesa, Sevilla.

en su conjunto un planteamiento radicalmente distinto del desarrollado con posterioridad. Este documento evidencia ser una propuesta de actuación planteada dentro del marco de una solicitud de concesión que Mengemor se disputa con El Chorro y sus poderosos accionistas, que contempla la construcción de dos presas semejantes y además ampliables (una estrategia obligada ante la inversión necesaria e indudablemente también planteada como aliciente de la oferta) pero no un proyecto técnicamente firme, respaldada eso sí por la solvencia y experiencia profesional del equipo que la redacta. Aunque Fernández-Shaw trabaja entonces junto a Mendoza en la Compañía Urbanizadora Metropolitana, en estos momentos permanece al margen del proyecto y será una vez se comience a trabajar sobre la opción de una presa con mayor envergadura cuando requiera de su intervención y lo incorpore al equipo (5).

\section{PROYECTO DE CONSTRUCCIÓN DEL PANTANO DEL JÁNDULA EN LA CHARCA DEL FRAILE PARA RIEGOS Y APROVECHAMIENTO DE ENERGÍA. 25 DE OCTUBRE DE 1925}

Este proyecto no es presentado por Mengemor, la disputa por la concesión con la compañía El Chorro ha sido resuelta y de su acuerdo surge Canalización y Fuerzas de Guadalqui- vir, sociedad que suscribe el documento. Si el de 1921 era una oferta técnica encaminada a la obtención de una concesión administrativa de dos aprovechamientos, el de 1925 es un proyecto desarrollado estrictamente para la construcción de un embalse con fines de riego y producción hidroeléctrica en el Jándula. Se trata de un documento con carácter semejante a un proyecto básico que define una presa radicalmente nueva y un salto ahora de $80 \mathrm{~m}$ cuya memoria se acompaña de unos anejos de diversa índole, tablas, cálculos y estudios varios y se completa con un juego de catorce planos y un pliego de condiciones.

El apartado referido a la Toma de agua es relevante porque muestra otro ejemplo de las variantes consideradas al respecto del diseño de la presa y de su aprovechamiento. Sabemos que dispone de tres tomas paralelas y centradas situadas a la cota +304 en el dorso de la presa, pero este proyecto preveía en cambio la ejecución de dos independientes de la presa y a alturas distintas para aprovechar los diferentes grados de llenado del vaso. La conducción hidráulica constituye otro de los elementos sustanciales del diseño que sufrieron una modificación ya que la propuesta anterior contemplaba un conducto a presión a través de la presa y en superficie tras superarla y acometer en la central y este, dos enormes túneles de sección circular y un diámetro de $3 \mathrm{~m}$ excavados en la 
roca de la falda opuesta de la cerrada, ya que por la derecha discurrirá ahora el aliviadero. La central tampoco ocupaba la posición centrada en el cauce y adosada longitudinalmente al cuerpo de presa, pues como puede comprobarse en la lectura del siguiente párrafo, era una preocupación de los proyectistas el proteger las conducciones hasta ella: «En el cauce del río hay grandes bloques procedentes de las laderas de la cerrada y demuestran lo peligroso que resultaría instalar una conducción al aire libre y atravesando la larga zona desde la presa a la central, sometida a los desprendimientos indicados. El trazado en túnel queda libre de estos peligros». Y para evitarlo sus autores podrían haber adoptado tan singular solución integrando en un cuerpo único presa y central, como una construcción robusta y protegida, al abrigo de los teóricos desprendimientos. Pero bien al contrario, prefieren alejarla con cautela todo lo posible de esta zona peligrosa de la cerrada. Observando el gráfico en el que se calcula la pérdida energética que ocasionaría comprobamos que su distancia estimada estaba en $300 \mathrm{~m}$. En cambio el plano titulado Perfil Longitudinal del Salto nos da otra información bien distinta por la que podemos concluir que la central estaría ubicada a casi $500 \mathrm{~m}$ aguas abajo y afirmar también que los autores aún no saben cómo podrá ser la construcción que la albergue (esbozada simplemente como la silueta de una casa) ni el lugar exacto que ocupará en la margen izquierda. Ningún documento gráfico ni escrito la describe desde el punto de vista constructivo pero citan como referencia la presa de Camarasa -levantada sobre el Ebro y concluida un año antes- con la que compartiría también otros aspectos organizativos del conjunto del sistema hidráulico además de la ubicación de la central y sus tomas hidroeléctricas.

Sí contemplaba que el túnel excavado en la roca se subdividiera en tres conductos de acero para alimentar los grupos hidroeléctricos de que constaría (dimensionados para un caudal de $8 \mathrm{~m} / \mathrm{s}$ cada uno y cuyo salto máximo neto sería aproximadamente de $80 \mathrm{~m}$ a embalse lleno produciendo una potencia de $7.000 \mathrm{cv}$ cada uno supuesto un rendimiento del 80\%). Excusan dar mayores detalles pues según afirman, «estos se fijarán de acuerdo con los fabricantes de la parte mecánica y eléctrica de la instalación e incluso es muy posible también que al efectuar el concurso para la adjudicación de esta maquinaria, nos veamos precisados a modificar accidentalmente estas características».

Se trataría pues de un documento para iniciar la andadura del proyecto. Baste decir que su ejecución requirió la elaboración de centenares de planos de todo orden, desde los generales de la implantación hasta los detalles más minuciosos que podamos imaginar de la maquinaria instalada en la central. Conscientes de ello, sus redactores tratan de mostrar un rigor suficiente que asegure la aprobación ministerial pero no pueden obviar las múltiples indefiniciones de que adolece «detalles accesorios» y por ello bajo el pretexto de acudir a especialistas en cada materia, posponen la concreción de muchos de los aspectos técnicos.

Es posible afirmar categóricamente que la central no se define en este proyecto de 1925 y probablemente sea así porque en realidad se barajan varias alternativas y entre ellas la novedosa posibilidad de situarla adosada al cuerpo de presa. Existirían lógicas reservas, los plazos acucian y una solución tan innovadora puede requerir muchas explicaciones de la Superioridad cuando lo que se pretende es conseguir una pronta aprobación. El Estado como garante de los intereses generales centra su preocupación en la capacidad del embalse salvaguardado el riego para las zonas agrícolas y la seguridad de la presa, el diseño de la central en realidad atañe a la sociedad concesionaria que debe ocuparse de ello por su propio beneficio. Puede que en semejante ambiente de dudas entre Mendoza y sus socios, y de confianza de la Administración, se entregara este proyecto ciertamente indefinido. El plazo establecido de seis meses, que incluso hoy nos parece ajustado, sería entonces mínimo si pensamos en los medios de comunicación, de cálculo o de representación disponibles. Ello condicionó no sólo el modo de presentación del proyecto, parcial, en etapas e incompleto, sino la toma de decisiones.

Pero el equipo continuará trabajando, momento en el que entre otras cosas se decidirá trasladar la central hidroeléctrica desde una margen del río hasta situarla sobre el cauce. Una posibilidad que en principio podría resultar extraña pero una vez considerada demostraría sus ventajas. El escaso caudal del río en verano no dificultaría su ejecución, en cambio el exiguo espacio en el seno de la cerrada para disponer con naturalidad la edificación o el peligro de desprendimientos que les obligada a alejarla en exceso y excavar las conducciones, eran inconvenientes indudables de mantener una ubicación lateral al cauce. Conseguir una expulsión de las aguas turbinadas en el sentido natural de la corriente, lograr un acceso indistinto a la instalación desde ambas márgenes, el ahorro consecuente en las conducciones -por su longitud y trazado como túnel en la roca- o la elección del lugar para situar la cantera de la que se extraería la piedra (en la margen izquierda), son razones que considero hicieron decantar definitivamente la balanza. Y en este momento entraría FernándezShaw para colaborar en su diseño (5).

En esencia se puede afirmar que lo destacable de este documento que ya no firma Mendoza sino genéricamente Los Ingenieros de Caminos, es que determina con bastante aproximación el que será el cuerpo de presa -con el paseo de coronación como puente entre las dos márgenes- pero ni sus mecanismos hidráulicos ni por supuesto su central, responden al diseño final.

\section{INTEGRACIÓN DE LA CENTRAL HIDROELÉCTRICA EN EL CUERPO DE PRESA. 1925-1926}

Este periodo es del que existen menos testimonios documentales pero resulta de importancia capital pues es durante estos meses cuando la presa se define verdaderamente y su central adquiere los rasgos particulares que la caracterizan y la hacen célebre. El Ministerio que en ese momento se ocupa de un notable número de estas obras hidráulicas distribuidas por todo el país (34 se finalizaron en el lustro 1925-1930) revisa el proyecto de Canalización y Fuerzas del Guadalquivir y estoy convencido lo hace en permanente comunicación con sus autores porque al informarlo (julio de 1926), varias de las objeciones que opondrán ya están siendo estudiadas. Y es que se sigue trabajando en aspectos fundamentales del diseño. La dificultad de prever con exactitud el volumen de agua en una avenida, el modo en que se presenta y cómo controlar su evacuación originó cierta incertidumbre sobre la solución más idónea para el aliviadero. Por ello el proyecto se enviará a Alemania para ser estudiado por Theodor Rehbock que en el Flussbaulaboratorium de la Universidad de Karlsruhe donde ensaya variantes elaborando modelos a escala de diversos trazados y secciones para el vertedero (6). 


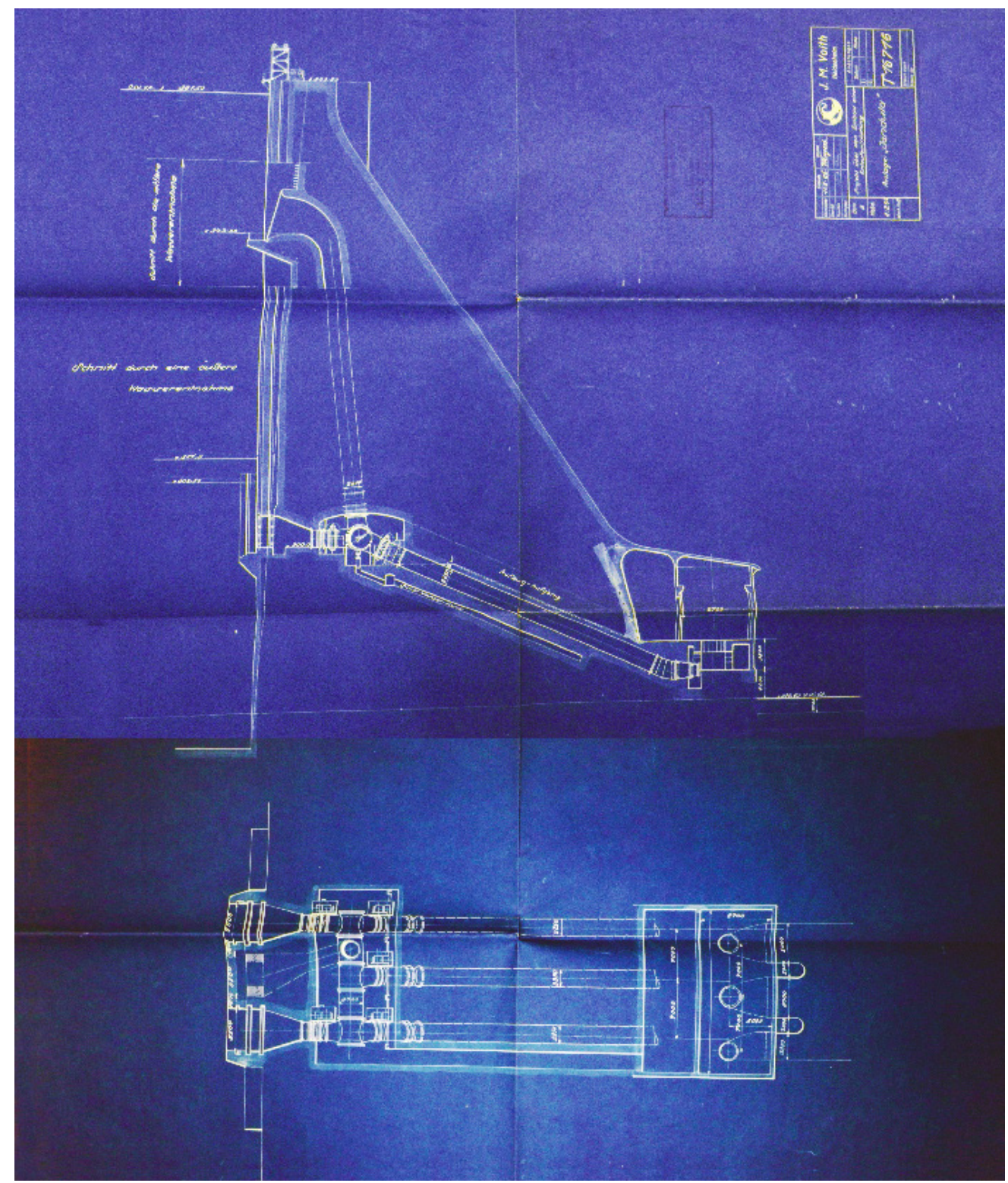

Figura 3. Plano T16716. E. 1:200. Fuente: Oficinas en el Salto del Jándula. Documento recuperado en la investigación.

Con probabilidad es en estos momentos también cuando Antonio del Águila Rada interviene como calculista en el proyecto. El equipo crece y va completándose con un nutrido grupo de agentes que aportan su conocimiento, sus técnicas o sus patentes industriales. Se tratará de una obra de enorme envergadura e importancia y por ello se establecen contactos con subcontratas para que colaboren, se inicia un nuevo modo de desarrollo del proyecto abierto a empresas nacionales y extranjeras, que trasciende el planteamiento inicial abarcado con un reducido grupo de personas afines a Mengemor. Empresas especializadas en los equipos para la central, del sector siderúrgico que suministre las conducciones o compañías que ofrezcan equipos auxiliares de obra.

Un hallazgo de la investigación es un plano de junio de 1926 suscrito por Wengerz como técnico de la compañía J.M. Voith (Figura 3) que propone unas tomas hidroeléctricas distribuidas en dos niveles intercomunicados sobre el dorso de la presa y una central ocupando la misma posición que la actual pero cuya organización, construcción y forma difieren de la línea de desarrollo proyectual que se seguirá más tarde. Se trata en este caso de una sala doble adosada a la presa, la mayor de ellas -cuyas dimensiones coincidirán con propuestas posteriores y ubicada sobre las turbinas- albergaría a los generadores y por su tamaño y posición podríamos suponer que la adyacente estaría destinada a los transformadores. No aparecen descritas todas las dependencias anexas de la instalación, ni tan siquiera la sala de control. Su construcción es visiblemente independiente de la del cuerpo de presa, no aparece como parte constituyente de él y su cubierta es de una ligereza tal que en ningún caso podría funcionar como trampolín de descarga ante un desbordamiento. Aunque su pequeño alero pudiera indicarnos eso (caso de la de Gaitanejo), la delgadez de su estructura impediría soportar cualquier paso de las aguas sobre ella. Sí pretende enlazar su volumen con el de la presa mediante el encuentro cóncavo del faldón con el macizo pero esa continuidad se limita al plano de cubierta y carece de naturalidad y dinamismo (5).

En esta propuesta el nivel de estiaje coincide con el proyecto de 1925 -lo cual podría confirmar su anterioridad a la variante estudiada con bóvedas- y la altura del piso en la sala de los generadores se establece a 7,80 m sobre este. En cambio en la 


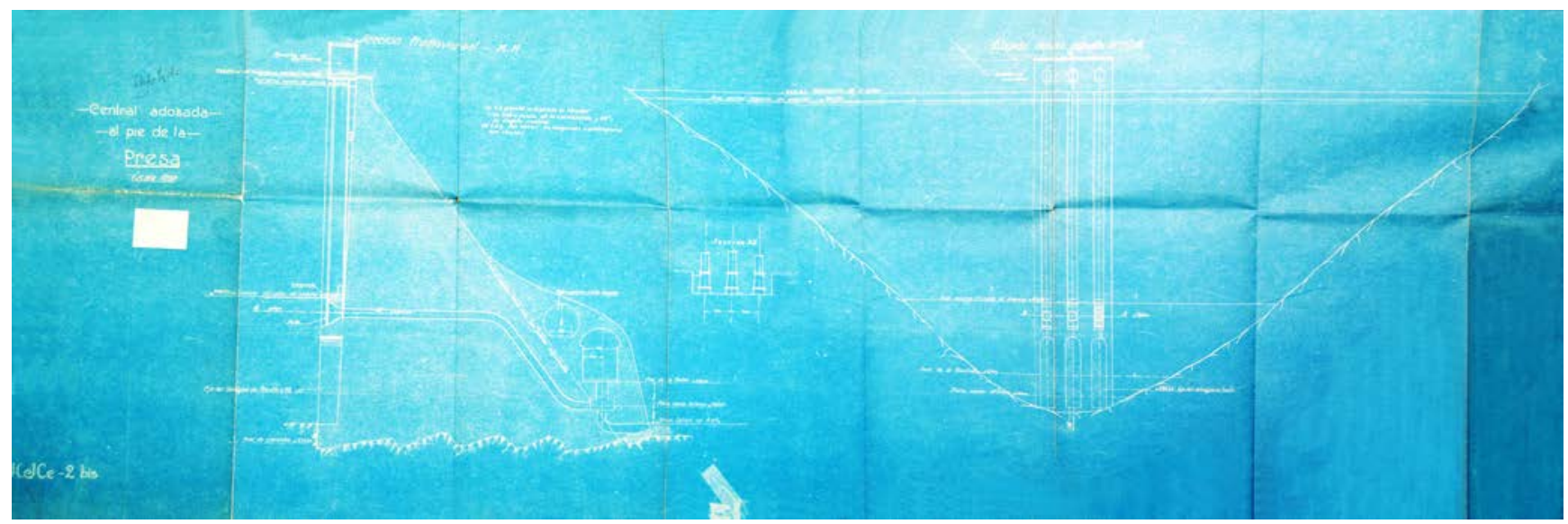

Figura 4. Plano JCe-2 bis - Central adosada al pie de la presa. E. 1:250. 1926. Fuente: Oficinas en el Salto del Jándula. Documento recuperado en la investigación.

idea que se desarrollaría a continuación -y más cercana a la realidad en su conjunto-, se fijó en la +279 m y la sala $10 \mathrm{~m}$ sobre dicho nivel. En cualquier caso sí coinciden ambas en el de las turbinas situadas a $4 \mathrm{~m}$ sobre el río. Desde la principal se planteaba una comunicación con la sala de válvulas (de anchura similar a esta pero de sección abovedada), una galería con la misma pendiente de las conducciones y dotada de un ascensor de cremallera. Su cuerpo de presa es más esbelto pero los niveles máximos del embalse son los mismos, lo cual requeriría su mayor curvatura. Probablemente esta solución quedara descartada por la inutilidad de una toma superior que quedaría sin uso frecuentemente y la complejidad del sistema en relación a la relativa ventaja de la reducción de las presiones de trabajo en las boquillas inferiores.

Es posible comprobar el debate en el seno del equipo acerca de las soluciones técnicas más convenientes y muestra de ello es esta multitud simultánea de ideas. De apenas unos días después de la variante mostrada, data otro plano trascendental, es el titulado Central adosada al pie de la presa (Figura 4), el primer documento en el que aparece la central en su configuración cuasi definitiva que he podido localizar (5). Una copia que no dispone de carátula y por tanto de fecha, pero sí presenta los sellos de registro con los que se identificaba cada documento (Entrada $n^{0} 652$ de 28 de julio de 1926. Salida no 658 de 28 de julio de 1926). Está identificado con el código JCe-2 bis y contiene un alzado aguas arriba de la presa, una sección por su eje y un detalle en planta de la embocadura de los conductos de las tomas hidroeléctricas. La sección, en la que aparece también por primera vez el torreón en la coronación (pero sin cubrir el paseo), muestra la disposición de las salas principales de la central acotando y referenciando los niveles del «piso» $(+289,00)$ y el plano medio de las turbinas $(+283,00)$, nivel este último que después variará. En una leyenda aclara: «las dos naves de máquinas e interruptores son rectas».

Dentro de la serie de dibujos elaborados en esta etapa preliminar a lo que apunta ser un verdadero proyecto de ejecución que ha variado nuevamente la geometría básica del macizo, existe otro significativo de un alzado de la presa. Está fechado en agosto de 1926 y su código es $J P r 14$ ( $J$. por Jándula y $\operatorname{Pr}$. por ser planos relativos al cuerpo de presa). Ofrece una revelación sorprendente al representar una envolvente diferente para las salas de la central hidroeléctrica. Su silueta dibujaría un arco conopial por lo que la cubierta de todas ellas, vistas hasta entonces solo en sección, no sería aún la que podíamos suponer (Figura 5).

Esta traza nos presenta una idea análoga para esta construcción adosada al faldón de la presa pero volumétricamente distinta y ante todo nos aclara que se contempla como un cuerpo único que alberga todas las salas y galerías frente al conocido conjunto de cinco cubiertas yuxtapuestas y de aristas perfiladas. Señala en el paramento los tramos escalonados con los que se adaptaría a esta figura pero no concreta su definición formal más allá de esta envolvente unitaria. El hecho de que no se aporte mayor información sobre cómo sería este diseño puede deberse a que se pretende en este caso definir las obras para la desviación del río y prever con precisión las de las tomas hidroeléctricas, o sencillamente que se desconoce todavía cómo será exactamente ese alzado de la central.

Y por eso otra de las copias del documento dibuja a mano una volumetría alternativa, esta vez fragmentada en tres. El cuerpo central -mucho más alto- podría dar cabida a las salas de los generadores y los alternadores en tanto los adyacentes albergarían los transformadores y dependencias anexas (Figura 6).

Como los anteriores dibujos, define los nuevos niveles establecidos para la utilización del embalse y de los elementos del cuerpo de presa. Sitúa las tres tomas, representa también seis juntas de dilatación dispuestas radialmente, señala los accesos de unas galerías de visita que nunca se ejecutaron y muestra los dos canales abovedados utilizados para la desviación del río durante la construcción y en cuyo interior se dispondrían los desagües de fondo previstos sobre la margen derecha (6).

A este respecto otras copias presentan unas anotaciones con lápiz rojo indicando una solución alternativa para estos desagües: marcan cinco salidas sobre la margen derecha y cuatro sobre la izquierda. Este hecho puede ayudar a datar todas estas tentativas pues la disposición de estos no se formalizaría hasta los primeros meses de 1927 y por tanto a afirmar que incluso a principios de aquel año, a pocas semanas del inicio de las obras, la central hidroeléctrica tenía una configuración en continuidad con el faldón de la presa, pero no precisamente la que se llevaría a término. Todas estas modificaciones nos muestran un nuevo modelo de organización de los sistemas hidráulicos y por ende del aprovechamiento hidroeléctrico. 


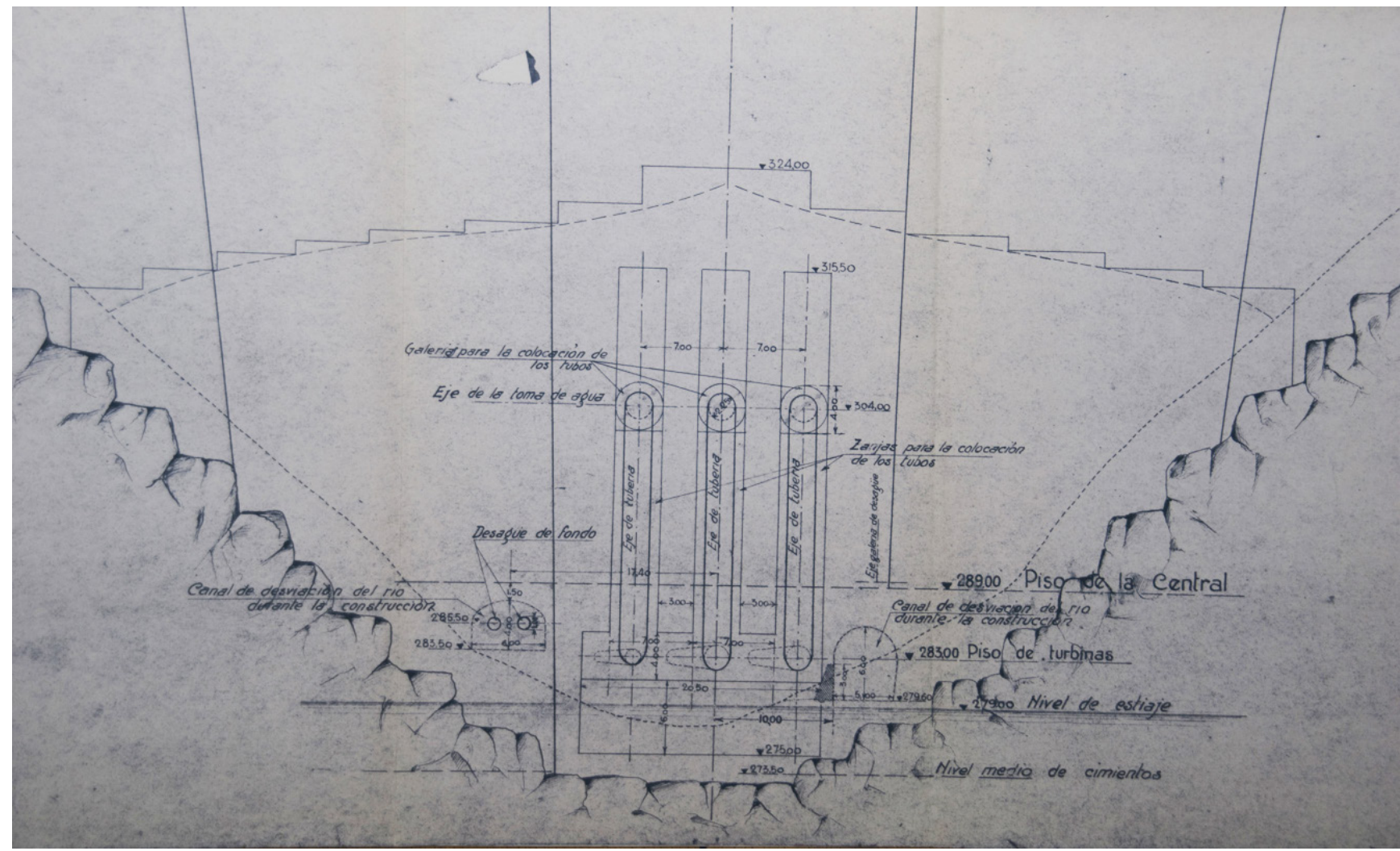

Figura 5. Detalle del plano JPr-14 - Alzado de la presa desde aguas abajo. E. 1:250. 1926. Fuente: Oficinas en el Salto del Jándula.

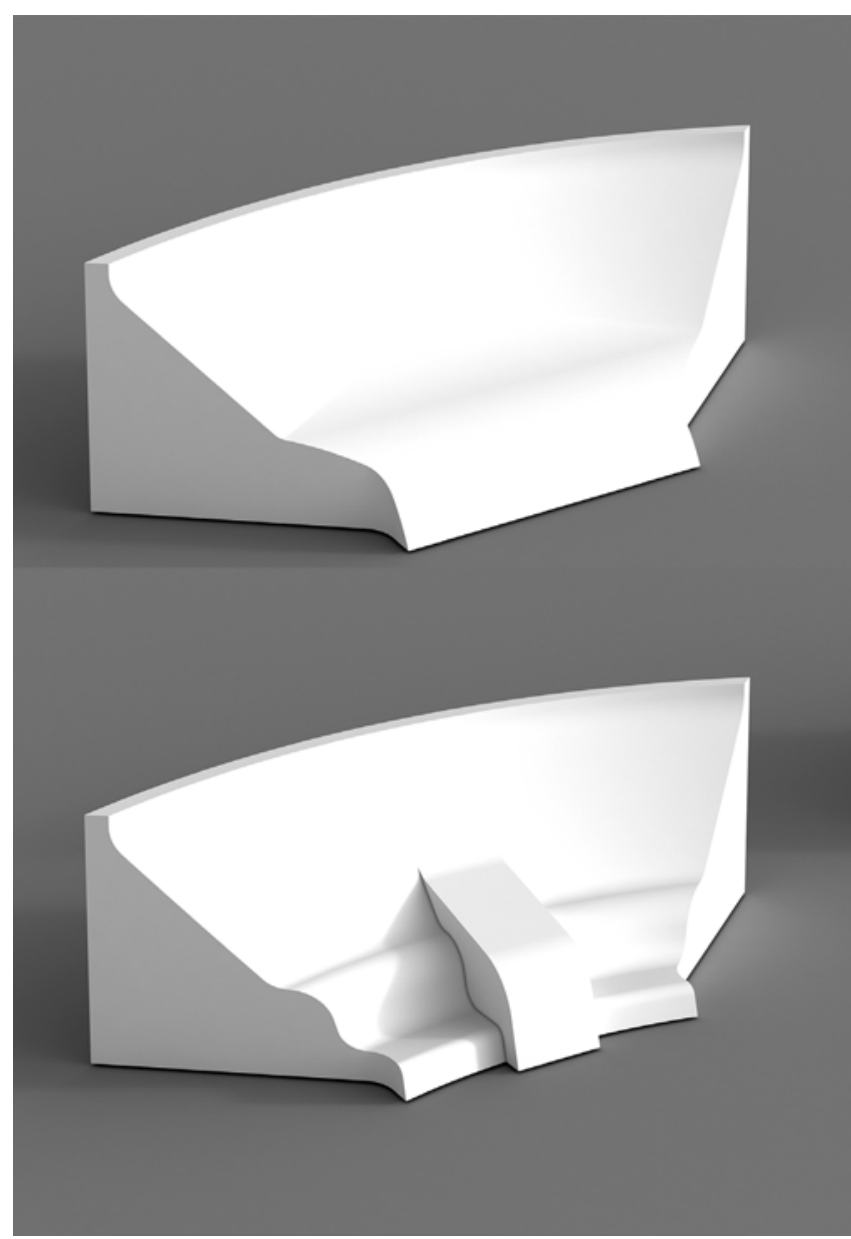

Figura 6. Recreaciones volumétricas de la central hidroeléctrica según el plano JPr-14 y su variante. Fuente: Nicolás Carbajal-Ballell.
La topografía del lugar encaminaba inexorablemente a una solución centrada sobre el cauce, necesidad que comienza a ser habitual por la elección de las mejores cerradas y se plantea en otros ejemplos con centrales al pie de la presa pero sin integrarse indisolublemente en ellas como este caso. Entre tanto y sin demora avanzan los trabajos previos en la cerrada donde ya se ha abierto una oficina y se inicia la contratación de mano de obra que alojarán en un nuevo poblado, el de La Lancha (7) (8).

De esta etapa existen dos dibujos a mano alzada de Fernández-Shaw (Figura 7). El primero cronológicamente es un apunte que dataría de los primeros meses transcurridos tras la entrega del proyecto de 25 ya que muestra una construcción de claras influencias expresionistas -más bien futuristas y en el que el influjo formal de la energía es patente- que sólo podrían haberse contemplado en los estadios iniciales de esta revisión general que se lleva a cabo y que puede vincularse con el plano de J. M. Voith. Sabemos hoy que este germen formal luego encontró un desarrollo proyectual diverso, por lo que si los primeros planos delineados en los que aparece definida la central hidroeléctrica que conocemos datan de mediados de 1926, este hermoso dibujo y las ideas que refleja necesariamente deberían ser anteriores. Se conforma con un cuerpo principal flanqueado por volúmenes redondeados que trata de establecer una relación -patente sobre el paramento de la presa- con el cuerpo de maniobras que se asoma desde la coronación y plantea una plataforma de asiento que no aparecerá en la planimetría del proyecto hasta bien entrado el año 1927 por lo que de nuevo es posible comprobar el debate continuo acerca de las soluciones más convenientes. El segundo de los dibujos muestra en cambio una presa muy similar a la ejecutada, en la que central y cuerpo de maniobras responden en esencia a la configuración final y en el que tan 


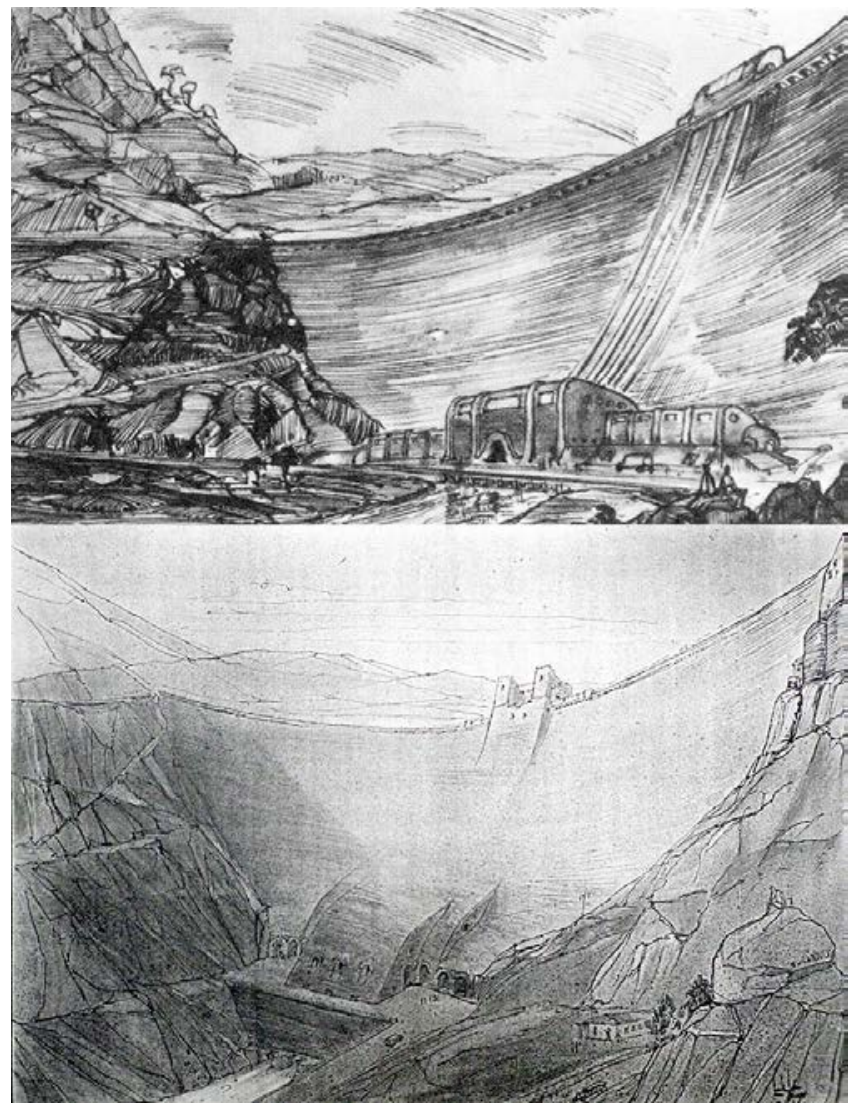

Figura 7. Dibujos de Casto Fernández-Shaw. Fuente: Revista ilustrada de información poética $\mathrm{n}^{\circ} 11 \mathrm{y}$ archivo de Endesa, Villafranca de Córdoba.

sólo la desproporción del seno de la cerrada -licencia quizá del dibujo- y el acceso de los vehículos -inviable en la práctica- lo alejan de la realidad (7).

\section{PROYECTO DE CONSTRUCCIÓN DEL PANTANO DEL JÁNDULA PARA RIEGOS Y APROVECHAMIENTO DE ENERGÍA PROYECTO DEFINITIVO. 1 DE FEBRERO DE 1927}

La real orden de 27 de julio de 1926 por la que se aprobaba el proyecto realizaba no obstante algunas observaciones que debían atenderse. Este nuevo documento venía a subsanar estos extremos. La memoria la firma únicamente Mendoza así como los planos adjuntos que en este caso son suscritos -como examinados- también por el Ingeniero Jefe de la División Hidráulica del Guadalquivir.

En lo que concierne al cuerpo de presa, modificaba su perfil y traza en planta como avanzaban los estudios de los meses precedentes y su ubicación se trasladaba unos metros aguas abajo a fin de favorecer una mejor colocación de las instalaciones auxiliares. El nuevo presentaba una curvatura mucho menor y lo hacía porque ya no era tan esbelto y no requería la colaboración geométrica del arco para soportar el empuje de las aguas. Como esa contribución estructural se obviaba, la presa se dividiría ahora en sectores independientes separados por juntas radiales.

El proyecto aportaba dos secciones, una por el eje de la presa que mostraba la que terminaría siendo la torre de maniobras sobre un paseo de coronación de $5 \mathrm{~m}$ e indica los niveles del embalse, las cotas de coronación $(+363,50)$, de las tomas de agua $(+304,00)$ y desagües de fondo $(+285,50)$, el nivel medio de cimientos $(+273,50)$, el de estiaje $(+279,00)$, el de la central hidroeléctrica $(+289,00)$ y la inclinación de los taludes del cuerpo de presa. La otra que corta por los desagües de fondo indicaba sus cotas de entrada y salida.

Pero la cuestión determinante que nos atañe de este proyecto es la reubicación de la central hidroeléctrica -recordemos muy alejada de la presa en el anterior-y ello se debe a la cuarta modificación recogida en la real orden: se adosará al pie de la misma y se colocará a eje. Esto conllevará el traslado de las tomas desde la falda de la montaña a la espalda de la presa y con ello el desplazamiento hacia la derecha de los desagües de fondo que ocupaban antes esa posición centrada. Pero el punto más destacado es la revelación que hacen en la memoria por la cual la forma de trampolín de descarga de la central hidroeléctrica se debe precisamente a esto, a preverla como elemento fluyente de las aguas desbordadas en una hipotética avenida durante las obras. Siendo así nada habría de metáfora, su perfil respondería al uso. Sí habría poesía, la que emana de la sutileza de sus formas. La previsión de que un desbordamiento pudiera acaecer repentinamente termina por dar forma a su singular perfil. Ello explica el distinto germen proyectual reflejado en los bocetos de Fernández-Shaw en los que se estudian sendas centrales hidroeléctricas y en la que sólo una de ellas -la desarrollada finalmente- permitía que las aguas rebosaran sobre ella. Uno sin mayor condicionante que el mecanismo hidroeléctrico y expresado como un enaltecimiento maquinista, el otro respondiendo a una función exógena y formalmente narrativo.

En este de febrero de 1927 se concreta la posición que adoptaría la central pero pese a lo que pudiera suponerse del examen de estos escuetos dibujos todavía no tiene la configuración definitiva. Posee tres cuerpos -no cinco como la construida- y el ocupado por las salas de máquinas irrumpía en el río para hacer fluir el agua directamente al cauce de forma que no era posible pasar frente a ellos hacia el otro costado de la presa. No existía la plataforma de asiento donde descansa actualmente la central, sus cuerpos laterales estaban al nivel de la sala de los generadores de manera que el de la margen izquierda funcionaba como acceso y el derecho albergaba en su seno la cámara de llaves de los desagües de fondo.

\section{FASE DE OBRA. DEFINICIÓN FINAL. 1927-1932}

Durante las obras aún se realizarían ajustes de su diseño. En marzo de 1928 podemos encontrar la primera documentación gráfica detallada de estos espacios. Hasta entonces habían aparecido escuetamente representados en alguna sección transversal figurando las salas principales, pero no es hasta este momento cuando puede considerarse que su compleja organización y singulares espacios quedan perfectamente definidos. Se trata de un juego de planos (JCe-24 al JCe-26) que a escala 1:100 representan en planta y sección el conjunto de salas, galerías y escaleras que la conforman. Sobre la plataforma de asiento se muestran los fosos para las llaves de los desagües de fondo. También aparecen ya definidos los tajamares que conducirían el agua turbinada o detalles como los raíles para mover los grupos, las canales para el cableado o conductos de ventilación.

Una sección longitudinal y siete transversales cortan cada una de las salas abovedadas junto a otras cuatro que seccionan la escalera de descenso a las turbinas, la sala de control y un detalle de la bóveda superior, completan la definición for- 


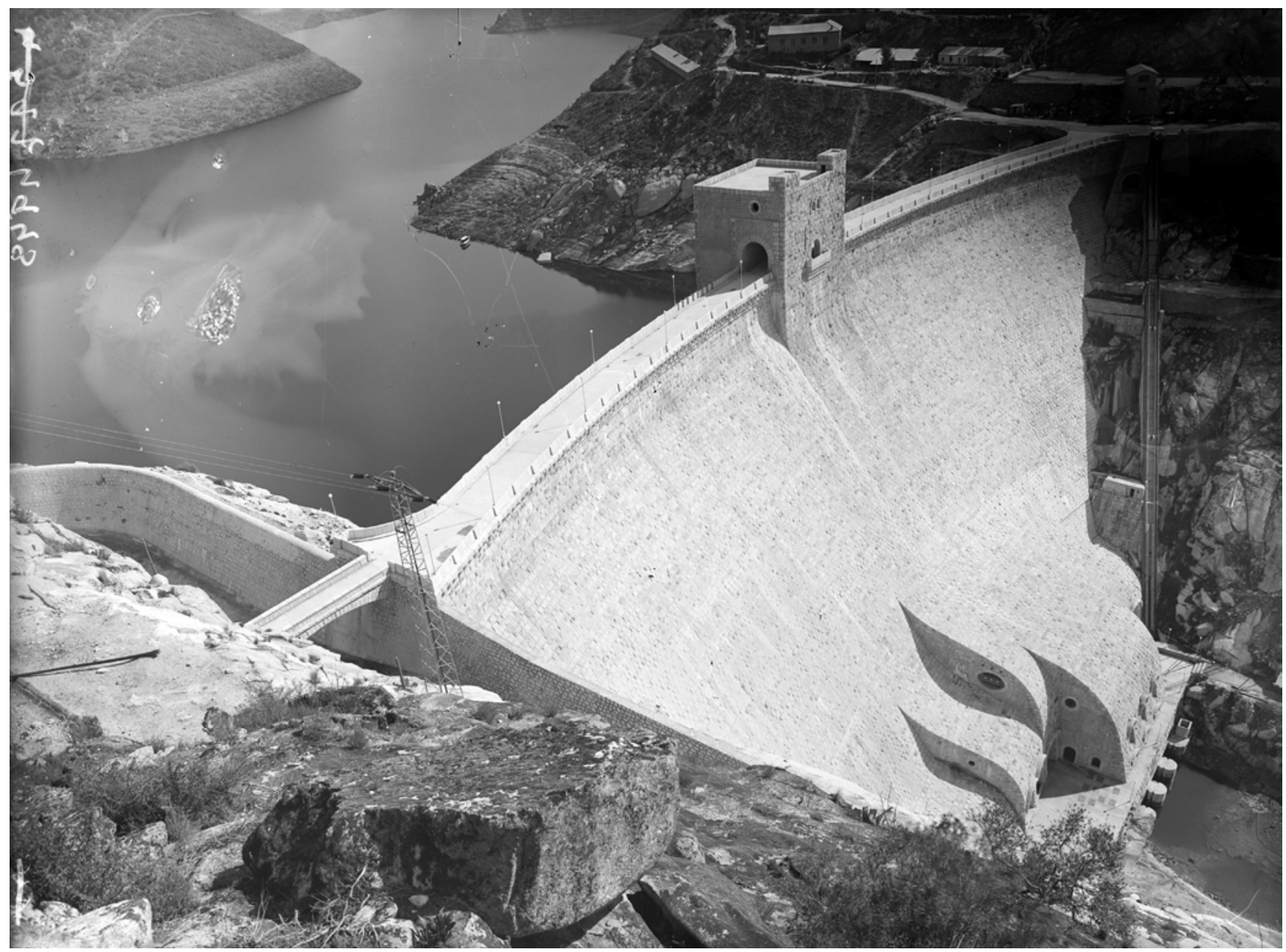

Figura 8. Salto del Jándula. L. Lladó. Fuente: (c) CSIC, CCHS BTNT.

mal de estos complejísimos al tiempo que hermosos espacios. Estas salas de inverosímiles secciones, están construidas en hormigón y revestidas al exterior por sillares de granito. Únicamente sus forjados intermedios son estructuras de viguería metálica. La central no está embebida ni parcialmente como afirman algunos autores (9), todas ellas respetan lógicamente el talud que constituye el paramento del muro de forma que se escalonan o adoptan la inclinación del cuerpo de presa. Estos dibujos no indican cómo se construirían, tan solo definen su geometría, pero su grado de exactitud hace suponer una fase muy avanzada de estos trabajos.

En verano se elaboran alzados y nuevos detalles de su intersección con el macizo, los huecos sobre sus paramentos, las hiladas y aparejos de piedra, las cornisas, molduras y demás elementos ornamentales o de las acometidas de la toma de agua y tuberías generales de refrigeración que le afectan, pero serán necesarios incluso los tres años siguientes con planos como el JCe-38. Interiores de la central (febrero de 1929), el JCe-62. Celdas 66.ooo voltios. (octubre de 1929), JCe-69. Pavimento de la sala de máquinas (enero de 1930) o con detalles de cerrajería como el JCe-73. Puertas de los transformadores de 1931 -posteriores incluso a su puesta en servicio- para definirla completamente (5).

\section{CONCLUSIONES}

Fueron necesarias sucesivas propuestas hasta alcanzar un proyecto -nunca definitivo- para la construcción de la pre- sa y su central hidroeléctrica. En ellos y en distinto grado intervinieron numerosos profesionales y empresas que conforman una autoría compartida de la obra. El modo en que se adjudicó - no se aprobó un proyecto de construcción estrictamente sino una concesión de un embalse con su aprovechamiento energético- permitió que fuera definiéndose progresivamente, con sucesivas reformas y modificaciones incluso durante las obras. Concretándose aspectos que hasta entonces no habían sido acordados o en ocasiones previstos y en estos planos de trabajo es donde podemos encontrar las decisiones específicas que lo definen. Donde verdaderamente se innova y profundiza en el estudio del proyecto y cuyos avances no siempre se recogen íntegramente en los documentos presentados oficialmente, recordemos simplemente cómo los aspectos constructivos de la central hidroeléctrica, desde la estructura de las bóvedas y forjados hasta su distribución interior y la definición de las fachadas o la simple elección de los equipos de producción, se definieron con posterioridad al inicio de las obras. Por eso no cabe hablar de un proyecto de la presa del Jándula si con ello tratamos de referirnos a un documento cerrado en costes, cálculos, definición formal, memorias y pliegos, firmado por un autor o autores en una fecha determinada.

La novedosa ubicación de la central hidroeléctrica adosada al cuerpo de presa se debe como se ha mencionado a la conjunción de los factores físicos relacionados con el lugar elegido, de los organizativos vinculados con la pla- 
nificación de la obra y de los económicos asociados a las alternativas planteadas. Podemos considerar que la idea proyectual que caracteriza esta central hidroeléctrica se planteó en el verano de 1926 y comenzó a desarrollarse a partir del denominado Proyecto Definitivo de febrero de 1927 al concebirse como elemento fluyente de un desbordamiento.
Desde entonces este Patrimonio Inmueble de Andalucía (BOJA 16-03-2006, $\mathrm{n}^{0}$ 51, p.54) ha sido ejemplo inspirador técnicamente, caso de la de Grandas del Salime en España o de las presas francesas de Chastang, Saint Etienne-Cantalés, l'Aigle o Génissiat (6)(10) -si bien estas con vertedero central- pero su inigualable originalidad formal la salvaguarda de imitaciones (Figura 8).

\section{REFERENCIAS}

(1) Gasset, R., et al. (1902) Plan Nacional de Aprovechamientos Hidráulicos (Plan Gasset), publicado por R. D. de 25 de abril de 1902.

(2) Martínez y Ruíz de Azúa. E. (1907) Plan de obras de riego de una zona de 95.0oo hectáreas con 40 por ciento de cultivo intenso en la región inferior del Guadalquivir.

(3) Mendoza, C. (1919) Proyecto de Canalización y Aprovechamiento de Energía del río Guadalquivir, entre Córdoba y Sevilla.

(4) García Redondo, N.; Gutiérrez Abad, A. (1996). Presa del Jándula y la canalización del Guadalquivir. Revista de Obras Públicas, $\mathrm{n}^{0} 3356$.

(5) Carbajal-Ballell, N. (2014). El salto del Jándula. Andújar, 1927. Casto Fernández- Shaw, Carlos Mendoza y otros. Colegio Oficial de Arquitectos de Almería.

(6) Carbajal Ballell N. J. (2015). El Salto del Jándula. Génesis de un paisaje (Tesis doctoral). E.T.S.A. Departamento de Proyectos Arquitectónicos, Universidad de Sevilla.

(7) Carbajal-Ballell N. (2016). El Salto del Jándula. Génesis de un paisaje. Ministerio de Agricultura, Alimentación y Medio Ambiente.

(8) Mendoza, C. (1928). Instalaciones auxiliares llevadas a cabo para la construcción de la presa del Jándula. Revista de Obras Públicas. $\mathrm{N}^{\circ} 2504$.

(9) Berrocal Menárguez, A. B., Molina Holgado, P., Cuvillo Martínez-Ridruejo, R. del (2017). Innovación en el diseño de centrales hidroeléctricas a principios de siglo XX. La ingeniería arquitectura de Casto Fernández-Shaw en la presa de La Jándula. Informes de la Construcción, 69(547): e206, doi: http://dx.doi.org/10.3989/ic.16.062.

(10) Gómez Navarro, J. L. (1940). Presas vertedero con central interna. Revista de Obras Públicas, $\mathrm{n}^{0} 2703$. 